Images du travail, travail des images

$2 \mid 2016$

Les ouvriers et la photographie : de 1945 à nos jours

\title{
Dominique Delpoux ou les visages du travail
}

Dominique Delpoux or the faces of labour

Jean Deilhes

\section{OpenEdition}

\section{Journals}

Édition électronique

URL : https://journals.openedition.org/itti/1211

DOI : 10.4000/itti.1211

\section{Éditeur}

Université de Poitiers

\section{Référence électronique}

Jean Deilhes, « Dominique Delpoux ou les visages du travail », Images du travail, travail des images [En ligne], 2 | 2016, mis en ligne le 01 septembre 2016, consulté le 27 juillet 2021. URL : http:// journals.openedition.org/itti/1211 ; DOI : https://doi.org/10.4000/itti.1211

Ce document a été généré automatiquement le 27 juillet 2021.

Images du travail, travail des images 


\title{
Dominique Delpoux ou les visages du travail
}

\author{
Dominique Delpoux or the faces of labour
}

Jean Deilhes

1 La représentation du monde ouvrier à l'heure actuelle, dans la photographie contemporaine, est loin d'être une question simple: elle fait ressurgir un sujet l'ouvrier - dont l'appartenance à la "classe ouvrière " parait dépassée ou révolue, et elle réactive une série de problèmes esthétiques qui n'ont cessé de traverser l'histoire de la photographie, documentaire ou non, notamment depuis son entrée dans l'ère du modernisme artistique au début $\mathrm{du} \mathrm{XX}^{\mathrm{e}}$ siècle. Les acquis de la dernière génération d'historiens du médium (Gunthert, Poivert, 2007), pour qui «cette compréhension neuve de l'apport fondateur des images d'enregistrement est le fruit d'une nouvelle histoire » (Ibid., 7), ne peuvent que nous encourager à interroger les rapports entre la « réalité » et ce que la photographie nous en donne à voir et à comprendre.

2 Pour tenter d'en saisir quelques-uns des principaux enjeux, nous articulerons la réflexion autour d'une série de vingt-quatre photographies réalisées par un artiste français en 2010. Conçues au départ dans le cadre d'une commande de "photographie d'entreprise ", ou corporate, ces photographies appartiennent au genre du documentaire. Mais très vite le contexte économique du rachat de l'entreprise Cofrablack par Orion Engineered Carbons (août 2011) et l'abandon de la commande conduisent l'auteur à réorienter son travail vers le champ artistique. Leur double nature mérite donc d'être examinée, en même temps qu'il s'agit de se demander précisément ce qu'elles peuvent donner à voir du monde du travail: une image conforme aux attentes sociales relatives aux représentations de l'usine, ou bien une " autre réalité » distincte des formes de représentations généralement adoptées?

Comprendre ce que ces photographies nous disent de spécifique sur le monde ouvrier, et tenter de réfléchir à la question de la représentation de la figure de l'ouvrier luimême, n'est possible ici, nous semble-t-il, qu'à condition d'appréhender ces images comme le fruit d'une démarche artistique qui s'inscrit pleinement dans l'histoire de la photographie. Car malgré l'apparente simplicité et la relative facilité avec laquelle tout 
spectateur peut identifier le genre (portrait), voire à en comprendre le sens en première lecture, leur principe d'organisation en série et leur appréhension comme un «tout » soulèvent divers problèmes d'interprétation dès lors qu'il s'agit d'en étudier la nature et le fonctionnement en tant qu'objet à la fois artistique et documentaire. Telle est leur ambiguïté fondamentale : ces photographies constituent par leurs fonctions descriptives très précises un document sur le monde ouvrier susceptible d'intéresser l'historien ou le sociologue, mais elles présentent aussi et surtout des caractéristiques formelles et matérielles destinées à les inscrire dans la catégorie des objets de l'art contemporain.

\section{Une question méthodologique}

Pour qui veut interroger les relations entre un médium comme la photographie et un sujet comme celui du monde ouvrier, il existe deux manières opposées d'entendre la question. La première entrée consiste à se demander dans quelle mesure et en quoi l'iconographie produite a contribué à forger notre imaginaire du monde ouvrier. Selon cette perspective, l'approche critique consiste à repérer des effets de discours dans l'image ${ }^{1}$, et à être attentif aux indices, variables selon les auteurs (Foucault, 1994) et dans le temps, qui font qu'une représentation, aussi soucieuse d'objectivité soit-elle, comporte toujours des marques de subjectivité de la part du photographe. Il faut se demander par exemple si l'intention de l'auteur consiste à valoriser le travail de l'ouvrier et ses gestes, ou, au contraire, s'il s'agit de sa part de dénoncer les conditions dans lesquelles celui-ci les effectue, en recourant à des procédés de construction de l'image destinés à souligner la présence d'un environnement confiné, oppressant, sombre, pollué, toxique, etc. Dans le cas de la photographie humaniste, pour prendre un cas tout autant emblématique de "l'iconographie nationale ${ }^{2}$ » que d'un moment de l'histoire sociale où les ouvriers occupaient une place centrale, chacun peut aisément relever comment les images parviennent quelquefois à pointer la fierté du regard d'un ouvrier saisi en plein travail, et entièrement préoccupé par l'effectuation de son geste, et se demander ce que signifie cette insistance à concentrer l'attention du spectateur sur les gestes ou l'action des mains sur l'outil, ou sur la machine, avec laquelle l'ouvrier fait corps ${ }^{3}$. S'agit-il, de la part de l'auteur de la photographie, de valoriser la figure de l'ouvrier? Comment cela s'inscrit-il dans les perceptions de la "classe ouvrière » et dans l'histoire des "Trente Glorieuses»? Cette imagerie rassurante quant aux conditions de travail ne masque-t-elle pas en partie la réalité de la «condition ouvrière » que s'efforce de décrire l'histoire sociale?

Il est aussi possible d'étudier l'usage des photographies dans l'espace éditorial du journal (Gervais, Morel, 2015), et de voir comment s'ajoutent aux langages de l'image des effets discursifs qui varient également en fonction des partis pris éditoriaux relatifs à des événements et à leur traitement journalistique. Ou encore de déplacer la question sur le terrain des pratiques artistiques et des « espaces discursifs » de l'art, pour parler comme Rosalind Krauss (Krauss, 1990). La représentation des mouvements sociaux en 1968 fournirait aussi une matière riche d'enseignements, sans parler des pratiques amateurs, des croisements entre photographie et cinéma (chez Chris Marker et Willy Ronis qui ont collaboré l'un avec l'autre par exemple)...

Bref, on le voit, il est tout à fait légitime de croiser histoire sociale et histoire de la photographie, notamment en ce qui concerne la question ouvrière; entre un médium 
qui prend son essor lors de la révolution industrielle au XIX ${ }^{e}$ siècle et celle de la classe ouvrière, il existe de nombreuses contiguïtés chronologiques y compris au $\mathrm{XX}^{\mathrm{e}}$ siècle. Entre un «art moyen » agissant comme un opérateur de distinction sociale (Bourdieu, Boltanski, Chamborédon et al, 1965) et l'approche sociale, les liens sont même à ce point évidents que l'on peut s'étonner du peu de littérature sur la question...

7 En revanche, pour qui veut questionner la représentation de l'ouvrier au sein des pratiques actuelles de la photographie (artistique ou non) et dans l'usage médiatique, les difficultés sont d'un autre ordre. En effet, la «classe ouvrière » est la plupart du temps perçue, dans les représentations collectives du moment, comme une catégorie obsolète, ou anachronique, avec laquelle les ouvriers eux-mêmes ne se reconnaissent plus de lien d'appartenance symbolique. Elle a disparu des « écrans » au fur et à mesure que la culture ouvrière, les bassins et les quartiers ouvriers semblaient quitter l'espace médiatique hexagonal au profit d'autres « réalités » comme celle du vote Front national (FN) ou celles des fermetures brutales d'usines (Gimel, 2011) ${ }^{4}$. La question ouvrière a migré, au gré des délocalisations d'usines, vers des périphéries où l'on la tient « loin des yeux, loin du cœur». Or cette disparition du paysage social est d'autant plus paradoxale, selon les études sociologiques récentes, que «les ouvriers constituent toujours le groupe social le plus important de la société française et que leur existence passe de plus en plus inaperçue » (Beau, Pialloux, 2012, 27). Ces perceptions, qui font de la figure de l'ouvrier celle de l'inactuel, vont en outre totalement à rebours des nombreux travaux de recherche récents qui montrent au contraire à quel point il est nécessaire et urgent de poser à nouveau la question des réalités du monde ouvrier, chez nous, en France, dans le contexte du capitalisme financiarisé.

C'est dire à quel point il est loin d'être évident d'aborder la question de la visibilité de la figure de l'ouvrier dans la photographie contemporaine. Faut-il pour autant chausser les mêmes lunettes pour étudier un corpus d'images produites à l'heure actuelle et pour questionner un fonds ${ }^{5}$ issu d'une période durant laquelle la "condition ouvrière " constituait une évidence sociologique partagée, un marqueur incontournable de la vie politique et dans laquelle les ouvriers formaient le premier groupe d'actifs ${ }^{6}$ ? (Eckert, 2006) Et comment des photographies pourraient-elles apporter quelque chose à une question aussi complexe que celle du monde ouvrier? "Une photographie est-elle vraiment en mesure de "révéler" quelque chose du travail ? Ou offre-t-elle avant tout une bonne occasion d'en parler? » se demande Henri Eckert (Eckert, 2012). N'y a-t-il pas une gageure à s'évertuer à faire parler des images totalement muettes quand c'est au contraire par une collecte de la parole ouvrière que cette réalité se construit?

Pour commencer à répondre à ces questions, du point de vue de l'histoire de la photographie tout au moins, il nous a paru opportun de retourner totalement le problème de ces relations et de se demander quels peuvent être les effets du sujet de la représentation en tant que tel - le monde ouvrier -, sur la manière de faire de la photographie (Lugon, 2001, 156) ${ }^{7}$. Quelle posture un photographe actuel œuvrant pour le marché de l'art contemporain est-il susceptible d'adopter face à un tel sujet et à quelles conditions celui-ci peut-il se faire « historien du présent »? En quoi le référent de la photographie, ici l'ouvrier sur son lieu de travail, est-il susceptible d'infléchir la manière de faire de l'image? Ou, pour le dire autrement, comment est-il possible aujourd'hui d'inscrire la figure de l'ouvrier dans le champ artistique contemporain?

Sur ce point précis, l'œuvre du photographe français Dominique Delpoux nous paraît constituer un exemple particulièrement intéressant des relations qui se tissent entre 
un sujet (l'ouvrier au travail) et le travail du photographe lui-même. Cette étude tentera donc de situer une des dernières séries réalisées par l'artiste en 2010 dans l'histoire de la photographie et dans l'œuvre du photographe, afin de montrer en quoi le portrait photographique de l'homme au travail est une affaire d'esthétique et d'éthique du documentaire largement tributaires, toutes deux, des enjeux et des débats qui traversent la photographie artistique depuis presqu'un siècle (Lugon, 2001) ${ }^{8}$, c'està-dire depuis qu'elle est entrée dans l'ère du modernisme photographique (Poivert, $2004 ; 2012)^{9}$. C'est à ce prix qu'il devient possible, nous semble-t-il, de comprendre le rôle et la place de la représentation de l'ouvrier et de voir comment de telles images de l'ouvrier peuvent être appréhendées comme des documents porteurs des valeurs du présent et susceptibles d'être interrogés par les historiens. S'il s'agit donc de décrire ici les conditions esthétiques et formelles grâce auxquelles la figure de l'ouvrier parvient à investir l'espace de l'art contemporain et à se voir conférer une visibilité que par ailleurs (photojournalisme et mass-média) elle semblait avoir perdue, l'enjeu est bien de saisir en quoi ces modalités, liées à la représentation, peuvent agir sur la perception des ouvriers photographiés ici.

\section{Une série de photographies}

11 Été 2010, zone industrielle de la presqu'île d'Ambès en Gironde, usine pétrochimique Cofrablack (devenue Orion), au cœur d'un site qui aligne pas moins de six entreprises classées SEVESO. Dominique Delpoux photographie une série d'ouvriers qui produisent le noir de carbone dont les particules ultrafines entrent dans la composition des pneus. Les images de ces « gueules noires » réactivent immédiatement les visages des mineurs dont la photographie noir et blanc a inscrit une trace indélébile dans les mémoires collectives. Les huit ouvriers retenus dans les prises de vues frontales ne forment cependant pas une galerie de portraits charbonneux simplement alignés les uns à côté des autres. Chacun a été saisi, lors de trois moments particuliers qui rythment la durée de son travail quotidien, à l'embauche et lorsque le travail prend fin, devant l'usine, puis enfin dans les vestiaires après la douche. La «journée » de travail durant laquelle s'effectuent les prises de vues, selon un protocole rigoureusement reconduit pour chaque ouvrier photographié, est à entendre ici comme une durée et non comme un moment diurne. Car les vingt-quatre portraits obéissent précisément au principe organisationnel des trois-huit auquel les «équipes » participent ici, photographe compris $^{10}$. La série photographique devient donc le résultat plastique produit par le principe du travail posté dans une usine où l'activité est continue. Chaque prise de vue en adopte le rythme et celui-ci fixe le "programme » dans lequel elles s'inscrivent, ce qui fait que les images ne prennent véritablement sens que dans la mesure où elles s'inscrivent dans un « tout».

12 Ces photographies d'hommes debout cadrés à mi-cuisse frappent par leur extrême netteté et leur réalisme rigoureux et franc. La lisibilité y est identique quelque soit le moment de la journée, la lumière additionnelle venant assurer une continuité parfaite entre les corps, d'un triptyque à l'autre mais aussi entre les trois vues frontales de chacun d'eux. Les valeurs tonales n'y changent donc pas - pour ce qui est du corps - du fait de la lumière ambiante, mais grâce à ce noir de carbone, qui, dans le panneau central des triptyques, s'est déposé tout au long de la journée sur les parties non protégées de la peau et sur les bleus de travail, ôtant tout effet de glacis à l'image. De 
part et d'autre les photographies qui flanquent l'image centrale, chronologiquement liées à l'avant/après, forment en réalité une variante d'un principe de reconduction duelle; et les deux états vêtus/demi-nus composent, en regard de celle-ci, une sorte de contrepoint dont la force est de permettre non seulement l'enregistrement du temps de travail et l'inscription des marques de fatigue, mais encore d'y faire varier on ne peut plus finement les attitudes, les poses, les uniformes et les positions des mains, l'expression des visages restant « neutre » mais pas identique. Les effets produits par la présence de ces ouvriers qui se dressent face à nous, obstruant la perspective du paysage industriel visible à l'arrière-plan, sont pluriels, complexes, et tout sauf univoques. Le rythme de la journée, perturbé et inhabituel, apparaît ici dans des variations subtiles, entre des extérieur-jour et extérieur-nuit, qui signalent que les heures d'embauche n'y sont jamais les mêmes pour chacun d'eux.

Triptyque 1. Série « Cofrablack 2010 ».
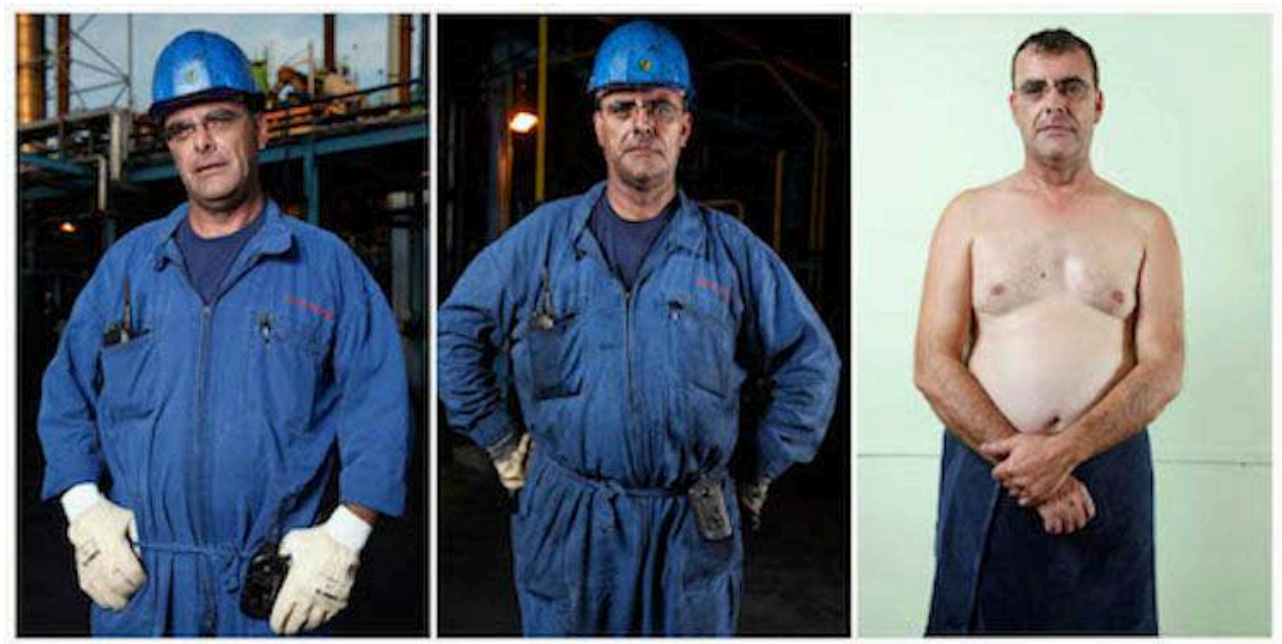

3 tirages chromogènes de $60 \times 90$ chacun

Dominique Delpoux, collection de l'artiste.

Triptyque 2. Série « Cofrablack $2010 »$.

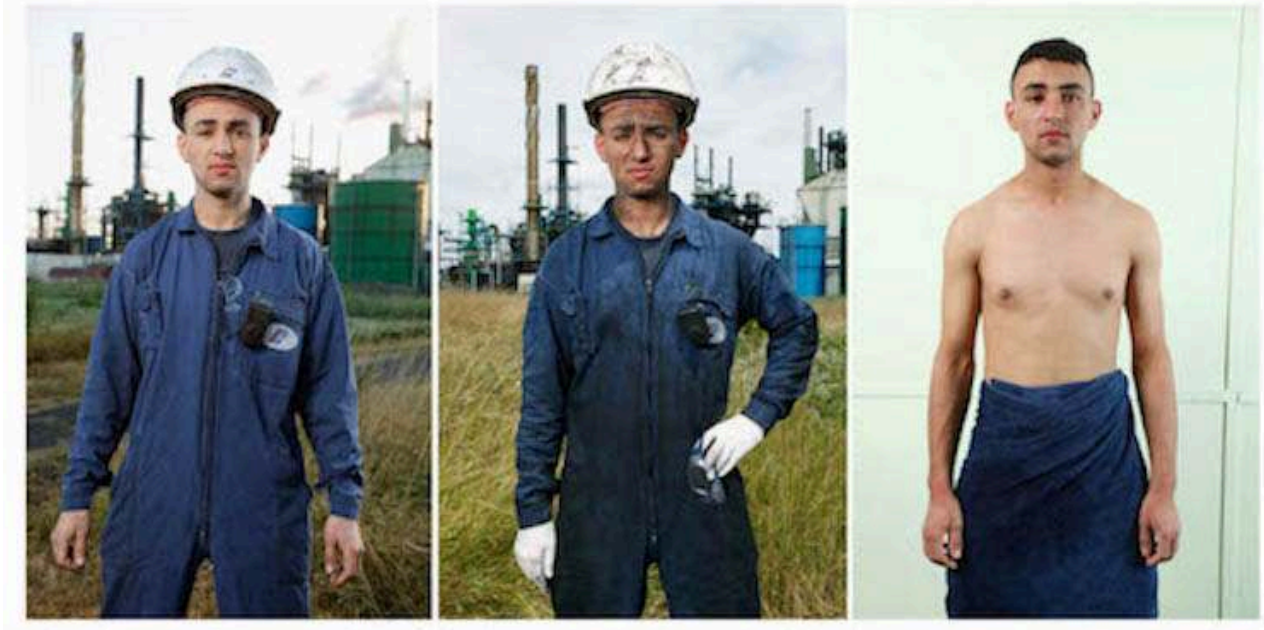

3 tirages chromogènes de $60 \times 90$ chacun.

Dominique Delpoux, collection de l'artiste.

Images du travail, travail des images, 2 | 2016 
Rien, dans le titre minimaliste des triptyques, ne semble devoir indiquer une quelconque relation avec des événements précis : il n'y a ici ni plan de licenciement ni menace directe sur l'emploi. Il ne s'agit pas plus de rescapés d'une catastrophe industrielle que "d'opérateurs » censés devoir célébrer les bienfaits d'une réussite économique du made in France. La frontalité de la pose confère au contraire aux personnels photographiés une expression essentiellement neutre. Le refus d'expressivité de la part de l'auteur semble obéir ici à un souci d'objectivité qui transparaît également dans les caractéristiques formelles des tirages, comme la grande précision du rendu, la qualité de la lumière et la gamme des couleurs naturalistes. Le format relativement grand des tirages, le genre du portrait et la disposition en triptyque méritent aussi d'être soulignés, comme autant de facteurs permettant de distinguer ces images de celles du photojournalisme et du photoreportage.

Ces portraits réactivent et mobilisent en quelque sorte des caractéristiques photographiques reconnaissables et déjà étudiées chez d'autres photographes du $\mathrm{XX}^{\mathrm{e}}$ siècle : le genre du portrait, mais aussi la question de la série, la frontalité de la pose et le regard dans l'objectif, ainsi que le "retrait» de l'auteur, qui est lié à la question de la neutralité dans la pratique du documentaire, forment quelques-unes des notions clefs, qui, dans l'approche historique du médium, peuvent faire sens et éclairer la démarche de l'auteur de la série des « Hommes de la Cofrablack ».

\section{Le genre du portrait entre document et art}

La référence incontournable, en matière de série de portraits photographiques, est incontestablement le photographe allemand August Sander auquel divers commentateurs de l'œuvre de Dominique Delpoux, à commencer par l'auteur lui-même, n'ont d'ailleurs pas manqué de faire appel. Dans les deux cas, en effet, l'on retrouve des photographies de modèles qui prennent la pose, explicitement, sans faire autre chose que de poser; ils ne sont pas saisis sur le vif, lors de l'effectuation d'un geste emblématique de leur tâche professionnelle, contrairement à ce que nous mentionnions plus haut pour le cas de la photographie humaniste. Mais la filiation avec Sander est loin de se limiter à ces quelques similitudes de pose. Ce fait mérite d'être expliqué. La réalisation des Hommes $d u X X$ e siècle, entreprise au milieu des années 1920 (Lugon, 2001, 19) ${ }^{11}$, constitue par son ampleur et sa portée - une « coupe » transversale de la physionomie d'une société toute entière ${ }^{12}-$, une œuvre à la fois colossale et exemplaire. De la frontalité de la pose des individus à la qualité descriptive d'images relevant de la "photographie exacte ${ }^{13}$ ", en passant par la typologie sociale et la dimension sérielle, cette œuvre éclaire à elle seule différents aspects et notions qui entrent en jeu dans l'approche du documentaire au $\mathrm{XX}^{e}$ siècle. Olivier Lugon a montré comment le projet encyclopédique de Sander s'est élaboré, sur le plan formel, au contact des « constructivistes figuratifs" de Cologne afin de "répondre parfaitement [...] au programme du groupe, à cette triple volonté d'incarnation de la structure sociale, de clarté et de neutralité » (Lugon, 2001, 71). Sa diffusion à l'échelle européenne commence, on le sait, avec la publication d'Antlitz der Zeit (Visage d'une époque) en 1929, sorte d'avant-goût de "l'œuvre complet» qui ne sera jamais publié du vivant de Sander. Les propos de Sander autour de $1931^{14}$ (Conrath-Scholl, Sander, 2009) permettent de voir que ses portraits "posés ", avec leur visée frontale, jugés en leur temps "démodés ", obéissent avant tout à un principe rigoureux qui les destine à 
appartenir à une série. Leur "valeur » ne peut pas apparaître seulement à l'échelle individuelle, puisque celle-ci repose explicitement sur l'adéquation de chacune avec le programme que s'est fixé le photographe :

"L'idée fondamentale de mon œuvre photographique Hommes $d u X X^{e}$ siècle, que j'ai entamée en 1910 et qui contient environ 5 à 600 photos, dont une sélection a été publiée en 1929 sous le titre Antlitz der Zeit ("Visage d'une époque »), n'est rien d'autre qu'une profession de foi dans la photographie en tant que langage universel et la tentative de dresser un portrait physionomique actuel de l'homme allemand prenant appui sur le procédé optico-chimique de la photographie, donc sur la pure mise en forme de la lumière. »

Il ne s'agit pas pour Sander d'une tentative de refléter " des » visages en tant que tels, mais plutôt, comme en atteste l'emploi du singulier «un» accolé au "portrait physionomique », de saisir, comme il l'affirme à nouveau dans la seconde partie de sa conférence radiophonique de 1931, « un portrait momentané de toute une génération et, en s'appuyant sur la physiognomonie, de la faire s'exprimer à travers des photos ». Sander croit pleinement dans les capacités de la série photographique à être intelligible: aligner des portraits «typiques des différents groupes qui forment la société humaine » permet selon lui d'atteindre le but qu'il assigne au médium :

«En résumant ce que nous avons dit sur la photographie, nous sommes forcés de constater que nous avons affaire à un champ particulier, qui obéit à ses propres règles et parle un langage absolument original. En voyant, en observant et en pensant, avec l'aide de l'appareil photo et en ajoutant une indication de date, nous pouvons fixer l'histoire universelle et, grâce aux possibilités d'expression de la photographie en tant que langage universel, influencer l'humanité entière. »

Si la visée principale du travail du photographe selon Sander est donc bien, on le voit, de se faire historien, celle-ci passe par une valorisation de la série. Or cette entreprise consistant à élever la photographie à un haut degré de réalisation conceptuelle et formelle a fait date. Désormais, nous dit Olivier Lugon, « c'est la qualité de l'ensemble sa cohérence thématique, l'ambition de son organisation, la force de ses mises en rapport - qui doit rejaillir sur ses constituants pour leur donner le poids et la signification artistique dont ils manqueraient sans lui. » (Lugon, 2001, 252)

Les avant-gardes artistiques de l'entre-deux-guerres constituent, au-delà de l'œuvre de Sander, des cas de figure tout à fait intéressants par lesquels s'opère la valorisation de la notion de série pour la photographie : en exploitant formellement les alignements géométriques des objets sériels produits par l'industrie, elles ont associé le travail industriel à l'idée de modernité en art. Nous leur sommes redevables, malgré les variantes idéologiques qui ont existé entre les constructivistes russes, les avant-gardes allemandes de la Nouvelle Vision et la Nouvelle Objectivité, et celles des artistes qui se croisèrent dans "l'échangeur culturel » parisien des années $1920^{15}$ (Chéroux, 2012), d'une certaine esthétisation de la machine, de l'usine, et de la production en série d'autant plus valorisées qu'elles se trouvaient enrôlées dans les référents du modernisme du XXe siècle $^{16}$. L'analogie entre un système technique de production industrielle et la machine photographique, qui permet elle aussi de produire des objets reproductibles, participe durant l'entre-deux-guerres d'une tentative de valorisation de la série et du médium de la photographie. 


\section{L'affirmation du « style documentaire » aux États-Unis} sentimentalisme des images délivre un message propice à la réconciliation d'un peuple avec lui-même. En outre, un projet comme celui de Sander ne "passe » plus après l'usage destructeur qu'ont fait les nazis de la physiognomonie et de la typologie sociale - nazis qui rejetèrent pourtant ce « visage » de l'Allemagne de Weimar des années 1920. Sont prisées des images davantage au service du texte, ce à quoi répond parfaitement la diffusion imprimée de la photographie. Les deux décennies d'après-guerre en France sont donc essentiellement marquées par un recul du documentaire tandis qu'en art ce sont des tentatives issues de la Subjektive fotografie initiées par Otto Steinert en Allemagne à partir de 1955 et centrées sur des expérimentations photographiques, de l'abstraction et d'un goût pour un graphisme exacerbé qui se font jour ${ }^{18}$. Surtout ce sont les valeurs associées à la subjectivité et à l'expressivité des auteurs qui priment sur celles d'une objectivité en laquelle on ne croit plus guère. Les pratiques du reportage et de l'illustration, liées à la question du documentaire, sont les seules susceptibles d'être rémunératrices pour les photographes; elles sont dissociées des pratiques artistiques qui pâtissent d'un marché inexistant ${ }^{19}$ et d'une diffusion bien moindre, les espaces d'exposition demeurant rares. 

dimension auteuriale, moins on trouve de neutralité dans l'image. Ou, pour le dire autrement, plus l'auteur s'efface et se met en retrait, moins l'on est enclin à mettre en doute la valeur documentaire de son travail. Selon ce principe, ou cette croyance, le gage d'une certaine objectivité reposerait sur une neutralisation des valeurs discursives, sur un effacement des marques d'engagement, et sur une négation des dimensions rhétoriques et expressives de l'image tels qu'on s'est même demandé parfois comment aboutir à autre chose qu'à une forme d'anonymat des auteurs si l'on poussait la logique jusqu'au bout. La fluctuation des valeurs du documentaire et des critères d'appréciation des auteurs, que ce soit dans les pratiques des photographes, dans la réception critique et institutionnelle de leurs travaux, dans les usages ou dans les discours théoriques, a connu de tels écarts et de tels retournements qu'il a été possible d'en faire l'histoire (Lugon, Poivert, 2006). Olivier Lugon rappelle à ce sujet que « dès son invention, le médium semblait en effet, à en croire les contemporains, né pour mettre à mal l'importance de ce créateur, défier sa capacité à marquer l'image de son individualité, voire à ruiner la possibilité même de l'attribution, exercice minimal de la définition de l'art en Occident et que les milieux photographiques ne cesseront de vouloir reconquérir. » (Lugon, 2006, 9) documentaire, selon cette perspective, comment a-t-il été possible, alors, de réconcilier valeurs descriptives, fonctions archivistiques ou testimoniales et singularité des auteurs ? L'histoire de la photographie durant les années 1980 fournit quelques repères sur les voies empruntées pour parvenir à une telle résolution. Il ne s'agit pas de refaire le récit de cette "conquête", mais de comprendre que l'on peut difficilement faire l'économie de réflexions méthodologiques dès lors qu'il s'agit d'évoquer des photographies qui possèdent à la fois, comme c'est le cas ici avec Dominique Delpoux, une fonction documentaire, des qualités descriptives, mais aussi des caractéristiques formelles supplémentaires telles que le neutre ou l'objectif y sont compatibles avec un critère d'originalité de l'auteur.

lesthétique documentaire, commune à toutes les portraits du photographe, est à replacer en particulier dans un double contexte de création artistique et d'institutionnalisation de la photographie, inaugurée en France en 1989 par l'exposition «Une autre objectivité » (Chevrier, Lingwood, 1989), durant laquelle s'opère et se diversifie la réconciliation entre formes documentaires et statut artistique du médium et dont l'aboutissement se situe dans la «forme-tableau ». « Le tableau est 
la forme et l'idée dans lesquelles s'incarne la description objective comme pratique moderniste. » (Poivert, 2010, 134)

Cette notion de tableau et ce bref rappel du contexte théorique et critique relatif aux photographies chargées d'une valeur descriptive offrent, nous semble-t-il, une grille de réflexion utile pour apprécier les photographies de quelques séries relatives à des portraits d'ouvriers de la mine, du textile, de l'industrie chimique ou des abattoirs produites entre le début des années 1990 et 2012.

Photographie 1. (Étienne et Cécile Walzack), série « Les Mineurs de Carmaux », 1992-1993.

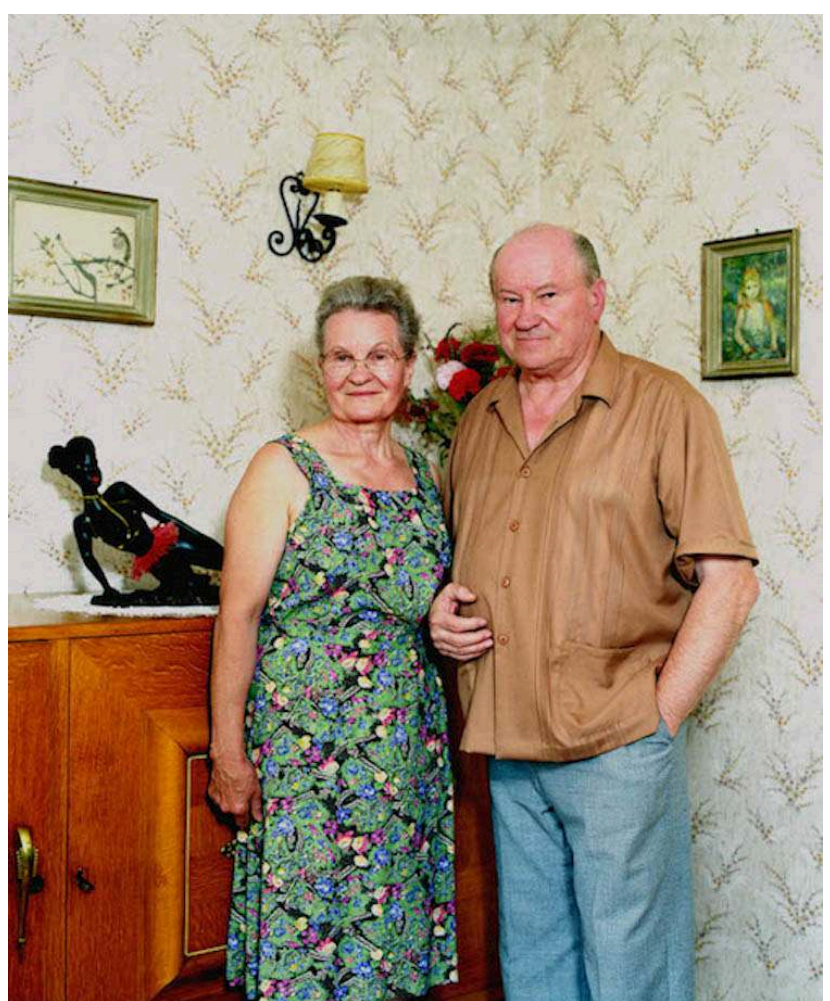

40 × 50 cm, tirages d'après négatif couleur, procédé « RA-4 » sur papier Kodak Endura Dominique Delpoux, collection de l'artiste. 
Photographie 2. (Marinette et Jean-Louis Fouques), série «Les Mineurs de Carmaux », 1992-1993.

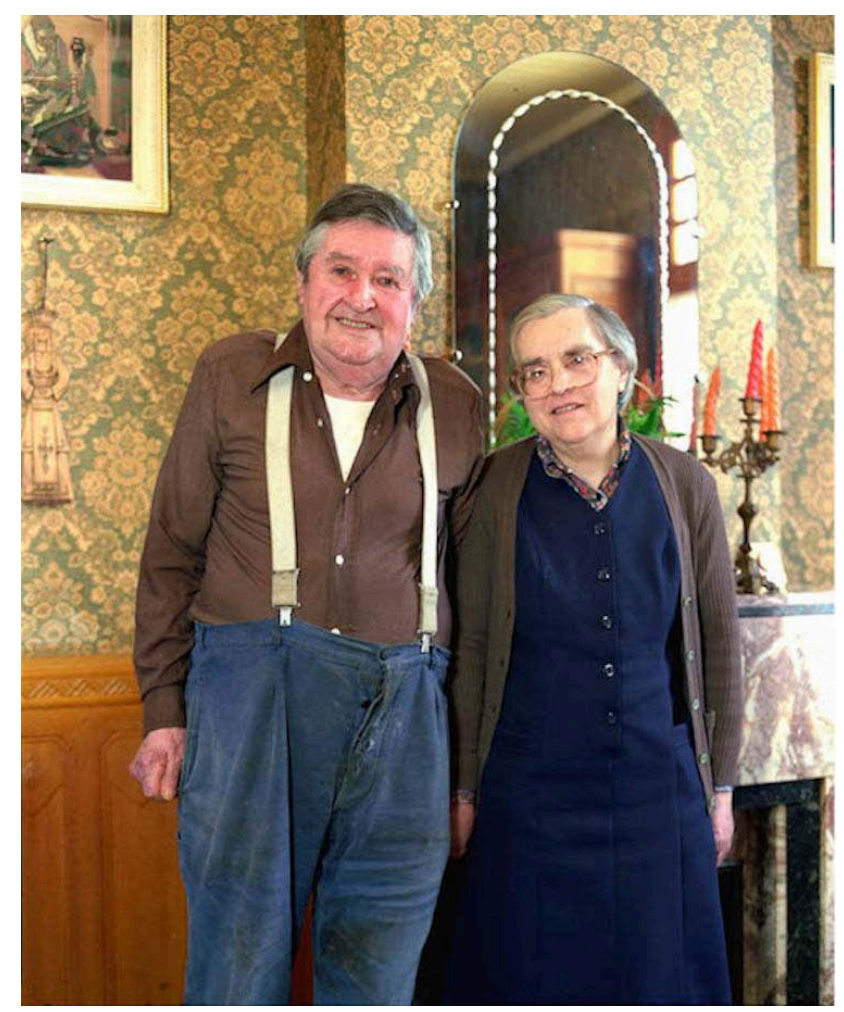

$40 \times 50$ cm, tirages d'après négatif couleur, procédé «RA-4 » sur papier Kodak Endura.

Dominique Delpoux, collection de l'artiste.

\section{L'œuvre du photographe Dominique Delpoux}

Dès la première série Les Mineurs de Carmaux en $1992^{20}$, rien de tape-à-l'œil n'apparaît : ni couleurs criardes, ni densité trop poussée, ni tons trop contrastés. Cette série semble à première vue obéir à une volonté de documenter un "corps social » en voie de disparition : l'ouvrier de la mine. Frontalité par rapport aux modèles qui posent, refus de la dramatisation des visages par la lumière et procédure objective correspondent ici à une " esthétique du neutre " parfaitement justifiée dans un tel projet. À la sagacité de son regard anthropologique correspond de fait une démarche profondément humaine ${ }^{21}$. On ne saurait employer le terme "d'humaniste », tant celui-ci, lorsqu'on l'accole à celui de "photographie ", se charge de significations particulières qui n'ont rien à faire ici. Pas de tendresse débordante comme chez Doisneau, pas de goût affirmé pour des lieux pittoresques, ni d'émerveillement pour les archétypes sociaux. À mille lieues du "réalisme poétique » de Cartier-Bresson, la photographie de Delpoux, lorsqu'elle donne à voir ces anciens mineurs qui prennent la pose, en couple, devant le buffet de leur salle à manger, n'est jamais nostalgique.

Certes, les trognes sympathiques des personnages qui peuplent les séries de l'artiste peuvent prêter à sourire au premier abord. Mais à y regarder de plus près, quelque chose d'autre apparaît à la surface de l'image. Cela provient d'un ensemble de détails infimes, qui semblent accorder entre elles les rides d'expression et les tenues vestimentaires et finissent par donner à ces couples un air de famille. Bien sûr, cette « consanguinité » n'est pas réelle, on se dit que ce n'est pas possible, et c'est peut-être 
là le coup de génie du photographe que d'avoir su laisser ce point à l'état de question. Est-ce à l'artifice de la photographie, et de la fixité à laquelle se trouvent soumis les modèles, leur visage, leurs mains et leur attitude qu'on doit cette ressemblance ? Cela provient-il de la pose elle-même ? Ou bien ces couples qui approchent de leurs noces d'argent finissent-ils par vraiment se ressembler, eux qui ont consenti au même quotidien, aux mêmes valeurs, aux mêmes aspirations et qui semblent appartenir au même bloc de destin?

Le propos est assez troublant tant il est vrai que ces couples constituent par leur longévité et par leur appartenance sociale au monde ouvrier des spécimens en voie de disparition; les fermetures des mines des anciens bassins industriels sont déjà loin. Mais on voit surtout comment le travail de l'artiste déplace la question du portrait vers d'autres territoires que ceux de la mémoire. En introduisant dans cette série la notion de double, de paire et de ressemblance, il conduit le spectateur vers d'autres usages que ceux de la valeur documentaire des photographies. Si «la dimension ethnologique et sociologique du portrait, reflet de l'intériorité " (Roegiers, 1994) est évidente, reste que le dispositif du diptyque, à savoir celui d'une photographie finalement conçue pour le mur des espaces culturels et artistiques de l'art contemporain ${ }^{22}$, nous invite aussi à une méditation silencieuse devant l'image, entre jubilation, tendresse et étrangeté.

Diptyque 1. (Anne et Thérèse), série « Les Jumeaux », 1995-1996.

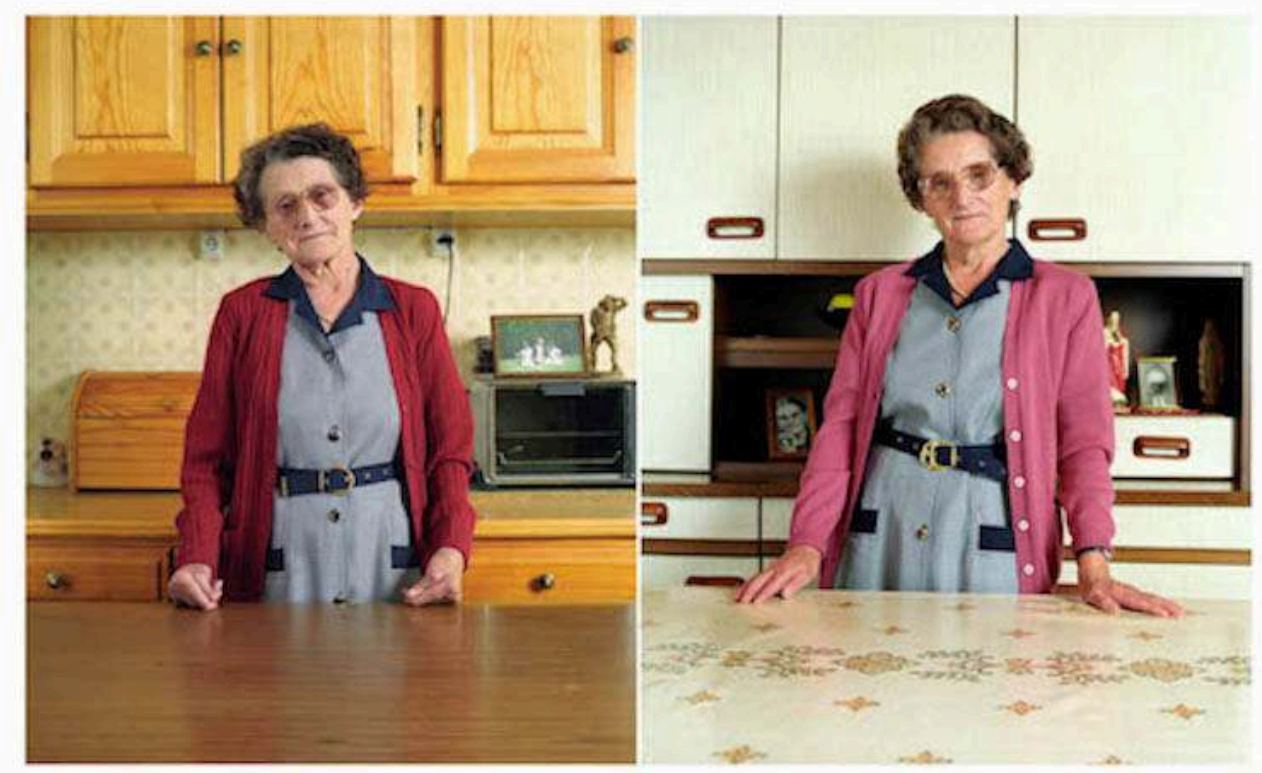

40 × 50 cm, tirages d'après négatif couleur, procédé «RA-4 » sur papier Kodak Endura.

Dominique Delpoux, collection de l'artiste. 

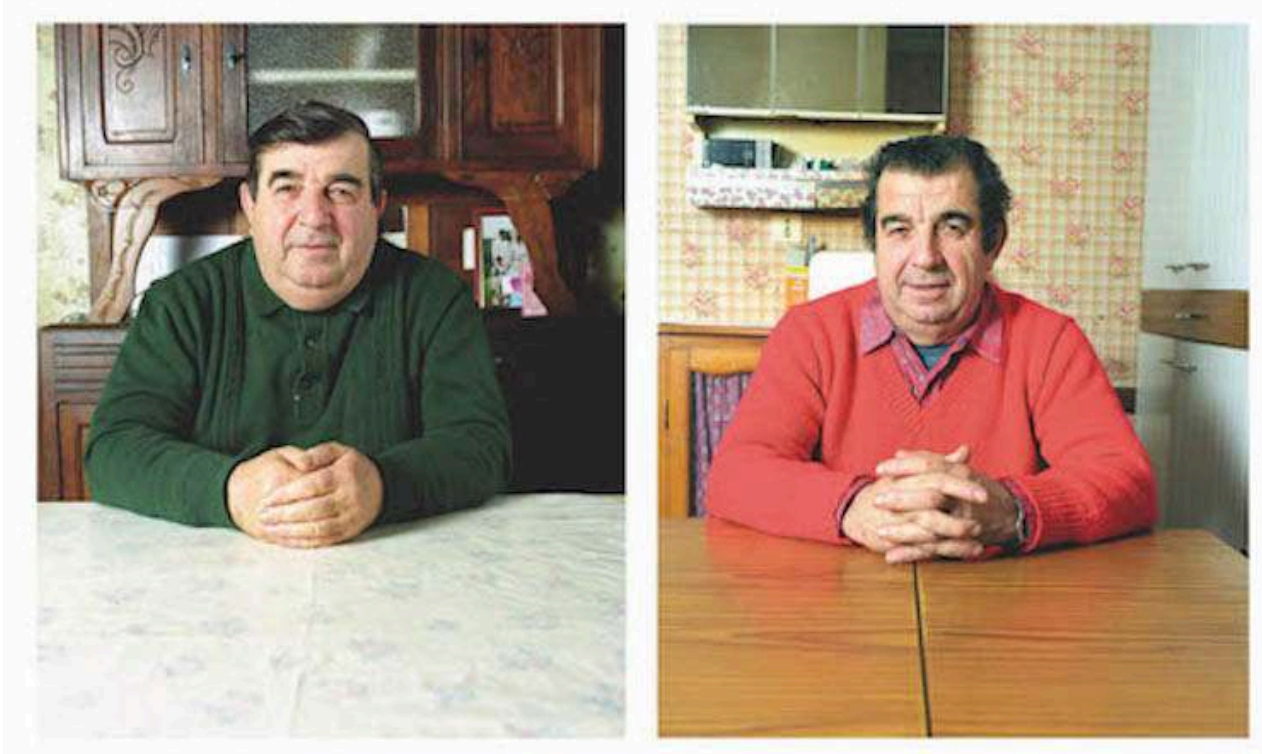

40 x 50 cm, tirages d'après négatif couleur, procédé « RA-4 » sur papier Kodak Endura.

Dominique Delpoux, collection de l'artiste.

Du point de vue de la question du double, la démarche de l'artiste semble prendre sa pleine dimension conceptuelle dans la série Les jumeaux en 1995. Ici, les paires de jumeaux qui font face à l'objectif se ressemblent vraiment, cela ne fait aucun doute. Mais on ne sait plus très bien parfois si cela provient des ressemblances du visage ou bien du décor et de la disposition des objets dans la cuisine qui offrent de troublantes similitudes. Dans l'une, c'est le pli dans la nappe en toile cirée d'un frère qui reprend la ligne médiane de la table en Formica chez son jumeau. Dans une autre, les téléviseurs et la hauteur du cadre au mur sont très proches. Rien ne permet vraiment de distinguer les deux visages, en tout cas pas la photographie qui fige la face des modèles. C'est un peu comme si le spectateur se trouvait invité au jeu des sept erreurs, tant les vêtements, le mobilier, le papier peint, la coiffure, le visage et l'attitude se répondent, lui donnant du fil à retordre pour s'arracher au trouble de cette ressemblance.

La photographie, médium de la reproduction «à l'identique » et en de multiples exemplaires à partir d'un négatif, se trouve ici mise en échec pour représenter fidèlement l'identité d'un visage. Le dispositif utilisé par Dominique Delpoux est à la fois simple et rusé. Il confronte cette machine à dupliquer l'image de quelqu'un à des êtres qui sont réellement dupliqués dans la vie, soit parce qu'ils ont un double (jumeaux), une moitié (époux), soit parce qu'ils sont saisis à deux moments de leur existence : matin et soir, au travail et au repos, avant et après le coiffeur, etc. 

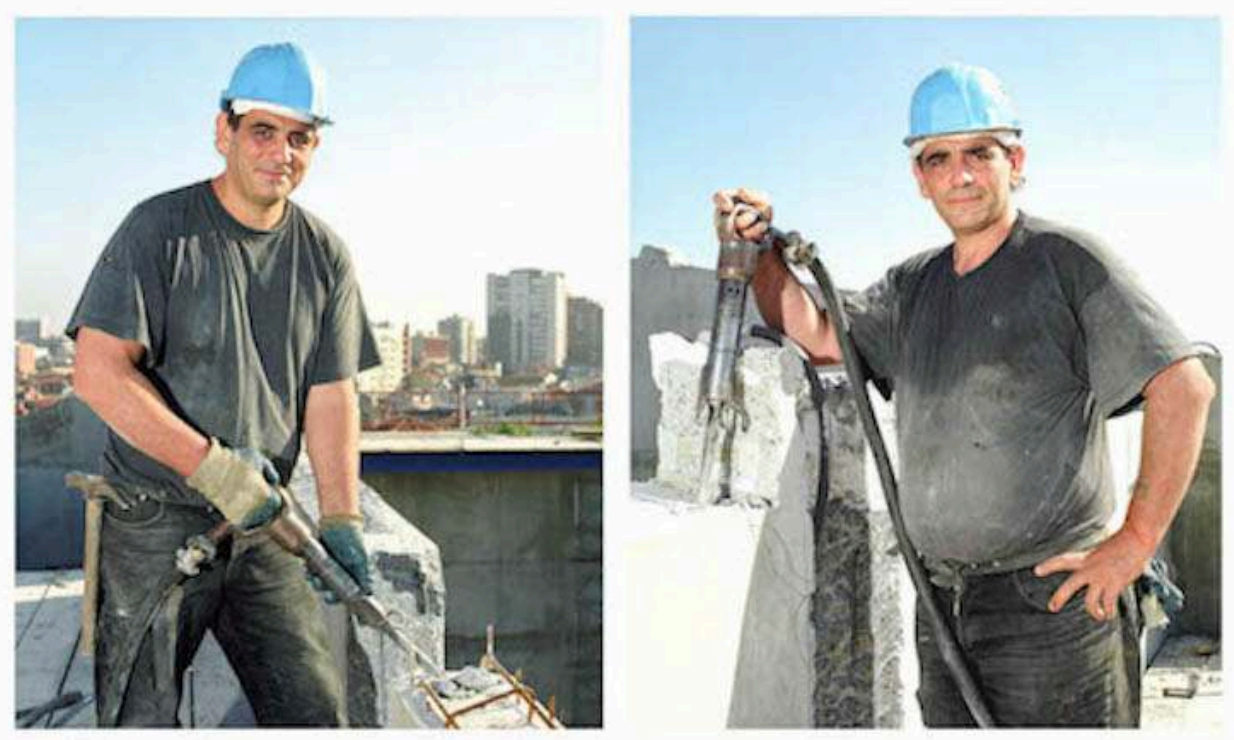

Théâtre national de Toulouse, tirages d'après négatif couleur.

Dominique Delpoux, collection de l'artiste.

La série des hommes du chantier du Théâtre national de Toulouse (TNT), réalisée dans le cadre d'une commande de son directeur lors de la construction durant l'été 1997, déplace le principe du double vers celui du temps, de sa durée et de ses intervalles, dans des diptyques où divers ouvriers sont photographiés le matin à l'embauche et le soir. L'introduction d'une dimension temporelle, dans la forme la plus simple du « avantaprès » et du «montage », conformes aux pratiques des reconductions photographiques sur les paysages urbains, déplace l'usage descriptif et patrimonial vers le concept abstrait d'une durée, celle de la dure journée de travail dont les visages, et leur portrait dédoublé, portent les marques d'une fatigue qui s'inscrit subtilement comme une trace indicielle. Mais le jeu de comparaison correspond aussi à une discrète mise en crise de la représentation: la valeur de vérité des portraits y est tout autant incontestable qu'elle se trouve remise en question, notamment parce qu'elle évite tout discours réformiste, toute orientation argumentative, valorisante ou non. Il s'agit de questionner l'efficacité du photographique, ici, et en particulier celle du genre du portrait, non d'asséner une idée sur le monde du travail ni de construire autre chose qu'une présence humaine au travail et, inversement, d'une inscription du travail dans le corps.

C'est avec la série intitulée « double Je » que Dominique Delpoux trouve à partir de 1999 une autre forme récurrente du double qui opère une sorte d'examen de l'identité professionnelle en vis-à-vis de l'image privée. «Chaque individu est présenté dans deux environnements distincts, objets d'un nouveau diptyque thématique. Le premier portrait est saisi dans le milieu professionnel, le second est mis en scène dans un cadre choisi par le modèle. Une vingtaine de représentants de catégories sociales diverses (pilote d'avion, artisan, président du directoire, avocat, ouvrier, professeur, femme de ménage, apprenti, artiste...) se prêtent à ce double jeu », indique-t-il lui-même. 
Diptyque 4. (Maryline, ourdisseuse), série « double Je », 1999-2003.
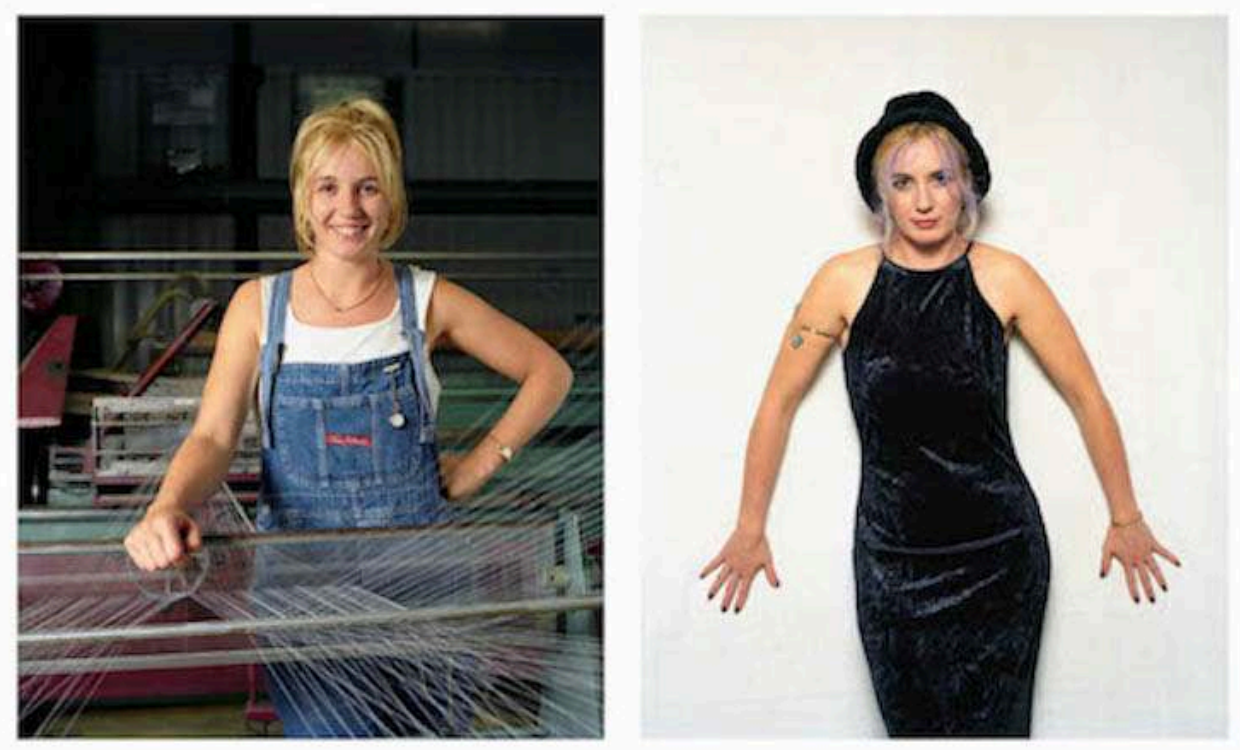

Tirage chromogène.

Dominique Delpoux, collection particulière.

\section{Sédimentation et complexification des principes esthétiques}

Ainsi est-il possible de voir en quoi la série des hommes de la Cofrablack (2010) condense et synthétise pleinement la majeure partie des principes formels apparus successivement depuis 1992 dans les propositions de l'artiste: dualité, similitude, gémellité, double, avant-après, public-privé... À l'échelle de chaque image, le potentiel descriptif des portraits est véritablement premier : le choix d'une frontalité de la vue, d'une lumière omnidirectionnelle, d'une netteté et d'une définition précise des contours et des matières en témoignent. Considérées de manière isolée, les photographies obéissent à des logiques descriptives poussées, dans lesquelles la « créativité » de l'auteur et la particularité singulière de son point de vue ne frappent évidemment pas le regard du spectateur au premier abord. Cela ne doit pas nous étonner, le photographe réclamant une sorte de filiation avec August Sander et Walker Evans, on l'a vu, qui se manifeste respectivement dans l'adoption du principe sériel du portrait et dans la reconduction des formes héritées de l'inventaire architectural.

Mais le recours à ce qu'il est convenu d'appeler un «style documentaire " ne peut se lire et s'interpréter sans la dimension matérielle (et sérielle) des « tableaux » qui n'ont pas l'archive comme horizon et dont le format relativement grand correspond à celui de l'exposition et de la collection. Ce n'est véritablement qu'à condition de prendre en compte le dispositif d'accrochage en diptyque ou en triptyque que les images sont susceptibles d'être appréciées et que leur portée réelle peut être mise au jour. De ce point de vue, le temps de la production de toutes ces séries est à replacer dans un moment de l'histoire de la photographie (d'art), les années 1990, qui correspond, pour Michel Poivert, à une refondation de la photographie au sein de l'art contemporain, refondation et non processus de différentiation comme catégorie séparée des autres 
pratiques artistiques. Le documentaire, tel qu'il fut remis au goût du jour, a permis de chercher des formes et d'opérer des propositions diverses dont l'effet fut, au minimum, de réactualiser le plaisir direct et immédiat de la contemplation du sujet et du référent dans les espaces de l'art - ici, notamment, la figure de l'ouvrier.

Dominique Delpoux bénéficie donc d'une période favorable à la photographie de type documentaire, et les distinctions obtenues par l'artiste dès 1994 avec son premier travail autour des anciens mineurs de Carmaux (prix Kodak de la critique photographique et lauréat du panorama européen de la jeune photographie professionnelle aux Rencontres d'Arles) montrent une œuvre significativement en phase avec les milieux artistiques et photographiques d'alors. Mais le soutien que le photographe reçoit en cours de travail de la part des institutions culturelles et artistiques régionales (Direction régionale des affaires culturelles Midi-Pyrénées : DRAC Midi-Pyrénées), et locales (Conseil général du Tarn), en 1993, montre aussi qu'il entretient d'emblée des liens avec les institutions culturelles et politiques : il adopte une posture à la fois professionnelle et artistique, dont il ne se départira d'ailleurs pas ensuite. Les nombreuses commandes, d'une part, mais encore les achats réalisés par le Fonds national d'art contemporain (FNAC) et les instituions à l'origine des mêmes commandes témoignent de l'inscription de l'artiste dans une posture peu autocentrée et pourtant extrêmement rigoureuse sur le plan esthétique et conceptuel. Il est possible d'affirmer que les liens tissés avec les instituions artistiques ont eu des effets réels sur son travail. Mais les apprécier du point de vue de l'histoire de l'art exige d'examiner comment les variations entre expressivité, objectivité, neutralité, engagement ou retrait, opèrent sur un fil extrêmement ténu, la juste distance par rapport au sujet relevant en quelque sorte d'un exploit. Le véritable exploit, c'est sans doute aussi le maintien de ce fil ténu d'une série à l'autre, alors que le photographe se trouve sur le fil du rasoir d'une commande institutionnelle sans arrêt susceptible de produire des effets de stérilisation du point de vue de la création.

La présence du sujet ouvrier dans différentes séries réalisées par Dominique Delpoux questionne donc bien le rôle et la fonction d'une représentation de l'humain au travail dans le champ artistique en regard du temps et du contexte culturel où elle se produit. Il est impossible d'affirmer, on l'a vu, qu'il y a de la part de l'artiste une volonté réformatrice; agir sur le monde via l'image n'est pas le propos, ici, et ses images ne visent aucunement à dénoncer des conditions de travail, à la manière d'un Lewis Hine ciblant le travail des enfants dans les champs de coton aux États-Unis dans les premières années du XX $\mathrm{XX}^{\mathrm{e}}$ siècle $^{23}$. Ni révoltés, ni emphatiques, ni portraits à charge, ni apologues, ses tableaux peuvent poser question voire modifier d'éventuelles représentations relatives au monde ouvrier chez le spectateur, bien que rien ne soit certain. Ce qui compte, c'est l'adéquation du résultat avec les espaces institutionnels dans lesquels et pour lesquels ces images sont réalisées, entre commande, aide à la création et achat d'œuvres, l'ensemble pouvant agir comme stimulant.

Contrairement aux images de Dorothea Lange, dont les talents de portraitiste lui ont valu d'être recrutée en vue d'accomplir une commande au sein de Farm Security Administration à la fin des années 1930, on ne trouve pas trace ici de « documentarisme compassionnel » (Poivert, 2010, 197). Cependant il est possible d'avancer que la reprise d'un tel «sujet » sur un temps relativement long, du point de vue de la pratique de l'auteur, correspond à une sorte d'empathie vis-à-vis d'une catégorie sociale, à tel point que l'on peut parler d'un système de valeurs. Photographier les classes populaires ne 
relève pas de l'exotisme social pour Dominique Delpoux, dont les origines tarnaises l'ont conduit en premier lieu vers la mémoire ouvrière du carmausin. L'identification et la visibilité conférées à tel ou tel ouvrier, dans des séries de portraits qui font appel à des caractéristiques formelles issues du style documentaire muries ou réactualisées à l'ère de la « forme tableau » et de la fusion art-document, n'est pas non plus «neutre » ou insignifiante. En effet, le format, la netteté, le refus d'une plasticité des arrières plans au profit d'un jeu plus discret autour des relations entre la figure et le fond, portées par des choix chromatiques rigoureux, en gros un souci de professionnalité photographique, semblent relever d'une autre identification, celle de la pratique de la photographie à un travail. Comme si au contact d'un sujet comme l'ouvrier, Delpoux se faisait lui-même ouvrier, un ouvrier particulier, certes, mais pas moins aux prises avec sa machine, ses réglages, ses lumières, ses fils électriques, et dont les gestes s'inscrivent également dans une chaîne d'opérations conduisant à une production. Ainsi, la question du travail dans la représentation se trouve-t-elle déplacée littéralement de l'extérieur du tableau, en tant que sujet ou référent de l'œuvre, vers l'intérieur du processus chargé d'en assurer la visibilité : le travail du documentaire.

Un tel déplacement de la figure de l'homme au travail vers le spectacle de l'image pour elle-même, s'il est en partie lié avec la destination de la photographie au marché de l'art et à la commande institutionnelle, n'est pas pour autant exempt d'effets sur les représentations du monde ouvrier. En montrant les huit ouvriers selon un parti pris formel rigoureusement identique, Dominique Delpoux parvient à s'appuyer sur les singularités physiques propres à chacun et à montrer comment les visages et les corps sont altérés par la nature du travail. Selon les âges et les particularités physiques, mais aussi en fonction de l'heure des prises de vues, le travail des ouvriers inscrit ses marques indicielles sur la peau et les corps. De ce point de vue la photographie, médium de l'indiciel, enregistre cliniquement la présence de cette poussière noire surfine sur la peau et suggère en passant la nature toxique de l'air qui a été respiré dans l'intervalle des huit heures de service. Le dépôt noirâtre sur le visage et les habits correspond ici à la partie visible du problème, il sert de révélateur discret, certes, mais néanmoins lancinant, de ce que les conditions du travail ouvrier dans les espaces industriels modernes de nos contrées proches méritent notre attention. Un détail comme la présence d'un petit boitier, fixé sur les bleus de travail, prend ici tout son sens : il s'agit d'un dispositif permettant de détecter la présence de gaz toxiques.

Mais le fait d'exposer de telles images dans les lieux de l'art contemporain a pour autre effet de rappeler ces faits dans des espaces et pour des publics que tout distingue justement des catégories ouvrières. Sans pour autant faire de la figure de l'ouvrier une sorte de clown subversif, ni un objet de divertissement, l'expérience proposée au spectateur, en 24 tableaux organisés en tryptique, procède d'un montage transversal grâce auquel les individus font ici " corps", au sens de corporation. C'est à dessein que le photographe opte pour la présence d'une uniformité des bleus de travail : marqueur iconique par excellence de l'appartenance à la "classe ouvrière", les individus présents ici, malgré les écarts générationnels et physiques, font bloc. Ils ne sont qu'un, ils sont les hommes de la Cofrablack. Mais l'identité ouvrière y apparait plus que jamais comme une question complexe: si les «bleus» et les visages noircis réactivent la mémoire ouvrière, la diversité des corps à moitié dévêtus replace les individus sur un terrain plus intime grâce auquel ces bustes masculins font pièce aux photographies des rugbymen $^{24}$ en nouveaux dieux du stade largement diffusés, ad nauseam, par les calendriers. Ces portraits offrent donc un contrepoint salutaire à ce que l'industrie 
médiatique produit comme modèles stéréotypiques du beau masculin. Ils confèrent à ces ouvriers une dignité et une présence très éloignées de « l'importante dévalorisation subie depuis quinze ans par les représentants ou les porte-parole ouvriers dans le champ médiatique. » (Beaud, Pialloux, 2012, 29)

Ainsi, l'hypothèse d'une certaine insolence pourrait caractériser le dessein du photographe : réintroduire la figure de l'ouvrier - mais un ouvrier moderne, dont les portraits n'autorisent aucune lecture physiognomonique destinée à en faire une type social particulier et défini -, dans les espaces institutionnels de l'art, ne relève-t-il pas d'une sorte de geste subversif? Car ces visages qui fixent l'objectif du photographe nous regardent dans les yeux, et nous impliquent particulièrement dans l'acte de réception des portraits : c'est un peu comme si leur condition nous « regardait». Or, au-delà d'un objet de contemplation offert aux spectateurs en général, cette série appartient bien aussi aux objets marchands de l'art et de la collection grâce auxquels les «dominants » pratiquent une spéculation financière et exercent (ou renforcent) leur position sociale distincte. Ces visages de «dominés » reviendraient donc, comme de véritables boomerangs, opérer un retour de la "condition ouvrière » dans les espaces symboliques qui travaillent le plus à les priver de visibilité.

\section{BIBLIOGRAPHIE}

Beaud S. et Pialoux M. (2012), Retour sur la condition ouvrière, Paris, Ed. La Découverte.

Bourdieu P., Boltanski L., Chamboredon J-C [et al.]. (1965), Un art moyen: essai sur les usages sociaux de la photographie, Paris, Ed. Minuit.

Chéroux C. (2012), « Du cosmopolitisme en photographie. Portrait de Paris en échangeur culturel », Voici Paris: modernités photographiques, 1920-1950 la collection Christian Bouqueret... exposition, Centre Pompidou, Galerie d'art graphique et Galerie du musée, du 17 octobre 2012 au 14 janvier 2013..., Paris, Centre G. Pompidou, p. 30-41.

Chevrier J-F. et Lingwood, J. (1989), Une autre objectivité / Another Objectivity, cat. exp., Centre national des arts plastiques, Paris / Centro per l'Arte Contemporanea Luigi Pecci, Prato, Milan, Idea Books.

Conrath-Scholl, G. et Sander, A. (2009), August Sander: voir, observer et penser [exposition, Paris, Fondation Henri Cartier-Bresson, 2009], Munich, Schirmer/Mosel, p. 28-29.

Eckert H. (2012), « Le chapardeur d'images (ou comment lire les images après les avoir dérobées ?) », Images du travail, travail des images, Rennes, PUR.

Foucault M. (1994), « Qu'est-ce qu'un auteur ? », Dits et écrits, tome I : 1954-1969, Paris Gallimard. Gervais T. et Morel G. (2015), La fabrique de l'information visuelle. Photographies et magazines d'actualité, Paris, Textuel.

Gimel J. (2011), Métaleurop. Mémoire ouvrière, mémoire d'ouvriers, Paris, Ed. L'Ours.

Gunthert A. et Poivert M. (dir.), 2007, L'art de la photographie, Paris, Citadelles \& Mazenod. 
Hamilton P. (2006), « La photographie humaniste : un style made in France? , La photographie humaniste, 1945-1968 : autour d'Izis, Boubat, Brassaï, Doisneau, Ronis [exposition, Paris, Bibliothèque nationale de France, Site Richelieu, Galerie de photographie, 31 octobre 2006-28 janvier 2007], Paris, Bibliothèque nationale de France, p. 26-33.

Krauss R. (1990), Le photographique : pour une théorie des écarts, trad. Jean Kempf et Marc Bloch, Paris, Macula.

Lugon O. (2001), Le style documentaire : d'August Sander à Walker Evans, 1920-1945, Paris, Macula.

Lugon O. (2006), « L'anonymat d'auteur » Le statut de l'auteur dans l'image documentaire. Signature du neutre - Documents 3, Paris, Ed. Jeu de Paume.

Poivert M. (2004), « La condition moderne de la photographie au XX $\mathrm{X}^{\mathrm{e}}$ siècle », L'ombre du temps : [exposition, Paris, Jeu de paume, 28 septembre-28 novembre 2004], Paris, Ed. Jeu de paume, p 14-39.

Poivert M. (2006), « Le document, au cœur du dogme moderniste », Le statut de l'auteur dans l'image documentaire. Signature du neutre - Documents 3, Paris, Ed. Jeu de Paume, p. 30-33.

Poivert M. (2010), La photographie contemporaine, Paris, Flammarion.

Poivert M. (2012), « La photographie contemporaine a-t-elle une histoire ? ", Photographiecontemporaine \& art-contemporain, Paris, Klincksieck, p. 75-82.

\section{NOTES}

1. La notion d'image est à entendre ici selon l'approche de Hans Belting pour qui les images «transitent entre les médiums historiques qui ont été inventé à leur usage. (...) Ce sont des nomades qui campent provisoirement dans chaque nouveau médium institué au cours de leur histoire, avant d'aller s'installer plus loin. » Voir Hans Belting, Pour une anthropologie des images, trad. Jean Bernard Torrent, Paris, Gallimard, 2004.

2. Il y a tout lieu, néanmoins, de nuancer cette dimension nationale pour la photographie humaniste. On lira à ce sujet la contribution de Peter Hamilton. Hamilton P. (2006), «La photographie humaniste : un style made in France? » dans, Beaumont-Maillet L, Denoyelle F. [et al.], La photographie humaniste, 1945-1968: autour d'Izis, Boubat, Brassaï, Doisneau, Ronis [exposition, Paris, Bibliothèque nationale de France, Site Richelieu, Galerie de photographie, 31 octobre 2006-28 janvier 2007], Paris, Bibliothèque nationale de France, p. 26-33.

3. Voir à ce sujet les photographies de Willy Ronis comme «Le ferronnier, 1945 », et de Paul Almassy « Ouvrier vérifiant une pièce », vers 1955, Paris BNF Ep-113 (1) Fol.

4. Voir par exemple l'enquête menée en 2008 par Josué Gimel suite à la fermeture de Métaleurop dans le Pas-de-Calais en 2003.

5. Nous pensons en particulier au fonds des archives du photographe Jean Dieuzaide à Toulouse sur lequel nous effectuons une recherche doctorale en ce moment. Ses portraits de mineurs dans le bassin de Carmaux et ses très nombreux reportages industriels réalisés pour la société HBA (Houillère du bassin d'Aquitaine) constituent des exemples emblématiques du traitement de la question durant les « Trente Glorieuses ».

6. Selon Henri Eckert, «c'est en 1975 que le groupe ouvrier avait atteint son essor maximal».

7. Selon la formule de la photographe américaine Berenice Abbot en 1940: "dans la photographie documentaire, le sujet de l'image contrôle dans une large mesure la photographie ». Cité par Olivier Lugon, p. 156.

8. Les histoires récentes de la photographie convergent en effet pour faire débuter la réflexion sur la question de la photographie documentaire dans le champ de l'art avec la figure d'August Sander, actif en Allemagne depuis 1910, date de l'ouverture de son studio à Cologne. 
9. L'ère du modernisme s'ouvre avec les figures d'Alfred Stieglitz et de Paul Strand aux États-Unis au milieu des années 1910. Mais le débat sur la question du modernisme photographique est beaucoup plus vaste et complexe qu'une simple question d'attribution.

10. Lors des entretiens que nous avons menés avec l'artiste durant le mois de novembre 2014, il est en effet apparu que celui-ci avait dû se prêter au rythme de travail des équipes qu'il intégrait en vue de réaliser les séries de 3 portraits. Sa présence dans les vestiaires n'était possible qu'à condition qu'il prenne part lui aussi à la douche collective.

11. Olivier Lugon identifie la première mention écrite de ce projet dans la lettre adressée en 1925 par Sander à son ami Erich Stenger.

12. En l'occurrence la République de Weimar.

13. August Sander préférait en effet cette appellation à celle de « Nouvelle Objectivité ».

14. Nous faisons référence ici au texte du tapuscrit original utilisé par Sander en 1931 pour prononcer sa conférence radiophonique diffusée en direct le 12 avril 1931 à la Westdeutscher Rundfunk de Cologne.

15. C'est la thèse proposée récemment par l'historien de la photographie Clément Chéroux. Voir Chéroux C. (2012), « Du cosmopolitisme en photographie. Portrait de Paris en échangeur culturel », dans, Bajac Q. et Chéroux C. (dir.), Voici Paris: modernités photographiques, 1920-1950 la collection Christian Bouqueret... exposition, Centre Pompidou, Galerie d'art graphique et Galerie du musée, du 17 octobre 2012 au 14 janvier 2013..., Paris, Centre G. Pompidou, p. 30-41.

16. Voir les photographies de René Zuber pour l'Exposition universelle de 1937, ou D’Arel Bauh, « mine », vers 1935.

17. Walker Evans a pris connaissance de l'œuvre d'Atget aux États-Unis grâce à la photographe Bénénice Abbot qui avait racheté le fonds Atget en France avant de retourner s'installer à NewYork en 1929.

18. Nos recherches en cours sur le cas du groupe "Libre expression", sorte de contrepoint français à la Subjektive Fotografie d'Otto Steinert, font apparaître cette dimension expérimentale et abstraite.

19. Jusqu'à la fin des années 1970 en France le marché de la photographie d'art reste à un niveau très bas même si les tentatives pour faire reconnaître culturellement et institutionnellement la photographie comme forme d'art se multiplient. La création des Rencontres d'Arles en 1971 en est un exemple.

20. Il s'agit de travaux de fin d'études, à savoir de la première série réalisée par le photographe.

21. Ce point de vue est conforme à la réception de cette série en 1994, primée aux Rencontres internationales de la photographie d'Arles, qui s'exprime chez le photographe Patrick Roegiers chargé des présenter l'exposition de la série : "sans indiscrétion ni voyeurisme, avec une proximité distante et une intense pudeur, [il] entérine l'identité physique de ces personnes anonymes, retirées de la vie active mais non de la société, qui incarnent la mémoire d'une activité révolue et qui affirment pudiquement leur tendresse ».

22. Cette série a fait l'objet en 1993 d'une aide de la DRAC Midi-Pyrénées et du Conseil général du Tarn, donc entre le moment des prises de vues et celui des tirages. La destination des images au champ artistique semble donc acquise.

23. Ce photographe, sociologue, a œuvré aux États-Unis au sein du Comité national pour le travail des enfants (NCLC) à partir de 1908.

24. Une des séries de Dominique Delpoux s'intitule d'ailleurs Les rugbymen. Lors de ce projet, que l'on peut rapprocher de celui du constat avant-après match de la série des boxeurs, l'artiste déconstruit ce type d'image de studio au profit d'une étude des variations des coups sur la surface de la peau, dans laquelle l'identité des sportifs se trouve photographiquement questionnée. 


\section{RÉSUMÉS}

La représentation du monde ouvrier dans la photographie contemporaine pose la question de la visibilité de la «classe ouvrière" aujourd'hui. Elle réactive aussi une série de problèmes esthétiques qui n'ont cessé de traverser l'histoire de la photographie, documentaire ou non, depuis son entrée dans l'ère du modernisme artistique au début $\mathrm{du} \mathrm{XX}^{\mathrm{e}}$ siècle. Pour tenter de le comprendre, notre réflexion s'articulera autour d'une série de vingt-quatre photographies contemporaines réalisées par l'artiste français Dominique Delpoux en 2010 dans le cadre d'une commande de "photographie d'entreprise " mais destinées à s'inscrire ensuite, grâce à une mise en forme particulière, dans le champ de l'art contemporain et de l'exposition. Cette double fonction, à la fois document et objet d'art, mérite d'être examinée en regard de ce qu'elle donne à voir du monde du travail et de ce qu'elle révèle du fonctionnement de l'image dans l'espace qui en organise la réception et la perception esthétique.

The representation of the world of labour in contemporary photography raises the question of the visibility of the 'working class' today. It also reactivates a series of aesthetic problems that have been continuously present in the history of photography, whether documentary or not, since entering the era of artistic modernism in the early twentieth century. So as to try and understand it, my reflection revolves around a series of twenty-four contemporary photographs by French artist Dominique Delpoux in 2010, which was part of a "corporate photography" order but was intended to belong to the realm of contemporary art and exhibition, through a subsequent specific formatting. This dual function, as both a document and work of art, is worth considering as it enables us to consider not only the world of labour but also the functioning of the image in the space organising reception and aesthetic perception.

\section{INDEX}

Mots-clés : documentaire moderniste, art contemporain, style documentaire, portrait, corporate photography, histoire de la photographie, Evans (Walker), Sander (August)

Keywords : modernist documentary, contemporary art, lyric documentary, portrait, corporate photography, history of photography, Sander (August), Evans (Walker)

\section{AUTEUR}

\section{JEAN DEILHES}

Jean Deilhes, (PRCE) enseigne l'Images et les médias à l'ESPE de Toulouse et l'histoire de la photographie à l'Université Toulouse Jean Jaurès au sein des départements d'histoire et d'histoire de l'art. Il est co-responsable du Master « Éducation aux médias et à l'information » à l'ESPE de Toulouse, et chercheur associé à la Galerie Le Château d'Eau à Toulouse. Dans le cadre de son doctorat d'Histoire de l'art, sous la direction de Jean Nayrolles (Toulouse II) et de Michel Poivert (Paris I), il étudie la photographie française durant les années 1960. 